\title{
Proposal of Evaluating Patients' Satisfaction about Quality of Healthcare System by Non-parametric Quality Control
}

\author{
Loc Nguyen \\ Sunflower Soft Company, Vietnam \\ Email: ngphloc@sunflowersoft.net
}

\begin{abstract}
Hospital quality assessment is key subject in hospital management. Patient satisfaction called criterion $F$ is an important quality measure. It is necessary to use $F$ as control factor to improve gradually the hospital management process. Because $F$ is fuzzy, I propose a so-called nonparametric quality control (NQC) method that is combination of non-parametric test and quality control chart in order to estimate $F$. If $F$ becomes a vector composed of many sub-criteria then, NQC method is extended as multivariate NQC (MNQC) method used for evaluating many concerned opinions of patients. MNQC replaces the distance between observation and null hypothesis by their Pearson coefficient. This research is a proposal because I do not make experiments on NQC and MNQC yet.
\end{abstract}

Keywords: patient satisfaction, hospital management, non-parametric test, quality control chart, non-parametric quality control, multivariate non-parametric quality control.

\section{Introduction}

The set of quality criteria (SQC) for evaluating hospital quality (Luong \& Nguyen, 2013) is issued by Vietnam Ministry of Health (MOH) is clear and it is easy to measure such SQC according to quality ranks. The term "hospital" indicates hospital, clinic, and healthcare system. As a convention, a period indicates day, week, month, quarter, or year. A short period indicates day or week whereas a long period indicates month, quarter, or year. With regard to the goal of serving patients by the best way, patients' satisfaction which is measured as criterion $F$ is important with note that $F$ can have sub-criterions. $F$ is called clinical satisfaction criterion. Because $F$ is fuzzy, every hospital should measure $F$ by itself. When a hospital reaches high $F$, it automatically meets SQC. However, it is not easy to estimate $F$ in every period because waiting for responses from many patients to a questionnaire consumes much times, which is discouraged task. Therefore, applying quality control (QC) approach into evaluating $F$ is an optimal solution. I propose a socalled $Q C$ process in which every hospital establishes a group of some officials called $Q C$ group who is responsible for asking a small enough sample of patients about their satisfaction ratings for hospital service in every short period. These ratings are used to determine $F$. After every long period, hospital's leader or KPI (Wikipedia, 2017) unit compares $F$ and criteria $A$ of SQC in order to find out causes of problems related to hospital service. If hospital's leader needs to determine a suspected factor which may be potential cause of lack of patients' satisfaction, $F$ is re-designed according to such factor. In other words, $F$ should be attached to criteria $A$ in SQC.

Section 2 is description of criterion $F$ and QC process whose core is non-parametric quality control (NQC) method. NQC is extended as multivariate NQC with complicated criterion $F$ in section 3. Section 4 is the discussion.

\section{Non-parametric quality control (NQC) method}


In this section, I describe how to measure $F$ by QC process. Because patients' ratings about their satisfaction do not conform normal distribution, I propose a so-called non-parametric quality control (NQC) method to realize the QC process. Suppose $F$ reflects the criterion A1.3 "The satisfaction of patients about examination process" (Luong \& Nguyen, 2013, pp. 1-6) in SQC. The patient response ranges from 1 to 5 where ratings 1 and 5 indicate "least satisfied" and "most satisfied", respectively. NQC method is an application of Wilcoxon signed-rank test (Walpole, Myers, Myers, \& Ye, 2012, pp. 660-663) into $\bar{X}$ control chart (Walpole, Myers, Myers, \& Ye, 2012 , p. 684). Let $X=\left\{x_{1}, x_{2}, \ldots, x_{n}\right\}$ be a small enough sample of ratings that QC group collects from $n$ patients for every short period as a week. Each $x_{i}$ may be associated with a cause $c_{i}$ that a patient explains her/his rating on hospital quality. Suppose patient ratings range from 1 to 5 and the acceptable satisfied rating is $\mu_{0}=\pi \approx 3.14$ which is an irrational number, for instance. Let $D=$ $\left\{d_{1}, d_{2}, \ldots, d_{n}\right\}$ be a set of deviations where $d_{i}=x_{i}-\mu_{0} \neq 0$. We assign a rank $r_{i}$ to each deviation $d_{i}$ without regard to sign of $d_{i}$. Let $R=\left\{r_{1}, r_{2}, \ldots, r_{n}\right\}$ be set of ranks. Let $W^{+}$be the sum of ranks whose corresponding deviations are positive according to equation 1 (Walpole, Myers, Myers, \& Ye, 2012, p. 660):

$$
W^{+}=\sum_{d_{i}>0} r_{i}
$$

Given $95 \%$ confidence degree, according to equation 2, the lower control limit $L C L$ of $\bar{X}$ control chart of $W^{+}$is (Walpole, Myers, Myers, \& Ye, 2012, p. 684):

$$
L C L=\mu-1.65 \sigma
$$

Where $\mu$ and $\sigma^{2}$ are mean and variance of $W^{+}$, respectively. Given $n \geq 15$, we have equation 3 (Walpole, Myers, Myers, \& Ye, 2012, p. 663):

$$
\mu=\frac{n(n+1)}{4} \text { and } \sigma^{2}=\frac{n(n+1)(2 n+1)}{24}
$$

After every long period as a month consisting of 4 weeks, hospital's leader or KPI unit receives 4 sums of ranks such as $\mathrm{W}_{1}^{+}, \mathrm{W}_{2}^{+}, \mathrm{W}_{3}^{+}$, and $W_{4}^{+}$. If there exists a value $W_{i}^{+}(i=\overline{1,4})$ that falls out the lower control limit $\left(W_{i}^{+}<L C L\right)$ then, the criterion $F$ is not satisfied. In this case, hospital's leader will survey all causes $c_{j}$ that patients provided in the $i^{\text {th }}$ short period ( $i^{\text {th }}$ week) in order to find out ultimate cause of lack of satisfaction. Otherwise, we conclude that patients are satisfied with the examination process of a given hospital with $95 \%$ confidence degree (1.65 sigma). When NQC method is implemented, the control chart and its lower limit can be improved so that they are more flexible. Viewpoint behind QC process is to consider hospital management as industry management. The QC process is repeated periodically until SQC is reached. As a convention, QC group, KPI unit, and hospital's leader are called users.

If randomization in survey is unintentionally violated, NQC method can be misinterpreted. So $F$ is fuzzy but it is essential to use $F$ as control factor for improving SQC with regard to ideology of Kaizen (Wikipedia, Kaizen, 2017) and 5S (Wikipedia, 5S (methodology), 2017). Another weak point of NQC is that we cannot ask patients in serious conditions such as critical injury, cancer treatment, and post-operation whereas processing such cases is preeminence of large hospitals. Therefore, the proposed QC process is most suitable to small hospitals and clinics in which most patients are outpatients. Larger hospitals should use $F$ as control factor to achieve SQC. Anyhow, $F$ is necessary because serious cases are processed likely well as patients with minor illness are satisfied.

\section{Multivariate non-parametric quality control (MNQC) method}


If $F$ is a vector of $k$ clinical satisfaction sub-criteria, $F=\left\{f_{1}, f_{2}, \ldots, f_{k}\right\}$ then, NQC method will produces $k$ control charts. Each chart together its lower control limit are used for controlling one sub-criterion $f_{i}$ but this solution is not optimal because users will get confused with many charts and it is difficult for them to decide whether $F$ is satisfied. I proposed a so-called multivariate nonparametric quality control (MNQC) method to solve this problem. MNQC method always considers $F$ as a unified vector. Recall that $X=\left\{X_{1}, X_{2}, \ldots, X_{n}\right\}$ be a small enough sample of rating vectors that QC group collects from $n$ patients for every short period as a week but each rating is now a vector $X_{i}=\left(x_{i 1}, x_{i 2}, \ldots, x_{i p}\right)^{T}$ where $x_{i j}$ is a partial rating value. Note, notation "T" denotes the transposition operation in vector and matrix. As a convention, the dimension of $X_{i}$ is $p$. The simplest technique for MNQC method is to define the deviation $d_{i}$ as the opposite of Pearson correlation between vector $X_{i}$ and the acceptable satisfied rating vector $\mu_{0}=\left\{\mu_{01}, \mu_{02}, \ldots, \mu_{0 p}\right\}$ according to equation 4 .

Where,

$$
d_{i}=-\frac{\sum_{j=1}^{p}\left(x_{i j}-\bar{X}_{i}\right)\left(\mu_{0 j}-\bar{\mu}_{0}\right)}{\sqrt{\sum_{j=1}^{p}\left(x_{i j}-\bar{X}_{i}\right)^{2}} \sqrt{\sum_{j=1}^{p}\left(\mu_{0 j}-\bar{\mu}_{0}\right)^{2}}}
$$

$$
\bar{X}_{i}=\frac{1}{p} \sum_{j=1}^{p} x_{i j}, \bar{\mu}_{0}=\frac{1}{p} \sum_{j=1}^{p} \mu_{0 j}
$$

As usual, we can set $\mu_{01}=\mu_{02}=\ldots=\mu_{0 p}=\pi \approx 3.14$. Like NQC method, we assign a rank $r_{i}$ to each deviation $d_{i}$ without regard to sign of $d_{i}$ after ignoring $d_{i}=0$. Let $R=\left\{r_{1}, r_{2}, \ldots, r_{n}\right\}$ be set of ranks. The sum of ranks $W^{+}$and the lower control limit $L C L$ are determined as usual (Walpole, Myers, Myers, \& Ye, 2012, p. 660) (Walpole, Myers, Myers, \& Ye, 2012, p. 684):

$$
\begin{aligned}
& W^{+}=\sum_{d_{i}>0} r_{i} \\
& L C L=\mu-1.65 \sigma
\end{aligned}
$$

Where $\mu$ and $\sigma^{2}$ are mean and standard deviation of $W^{+}$, respectively. Given $n \geq 15$, as usual we have (Walpole, Myers, Myers, \& Ye, 2012, p. 663):

$$
\mu=\frac{n(n+1)}{4} \text { and } \sigma^{2}=\frac{n(n+1)(2 n+1)}{24}
$$

If $W^{+}$falls out the lower control limit $\left(W_{i}^{+}<L C L\right)$ then, the criterion $F$ is not satisfied.

An alternative technique is to apply the classical parametric Hotelling's $T^{2}$ test into multivariate sample in which $d_{i}, r_{i}, W^{+}$are now vectors. Let $d_{i}=\left\{d_{i 1}, d_{i 2}, \ldots, d_{i k}\right\}$ be deviation vector between $X_{i}$ and $\mu_{0}$ where $d_{i j}=x_{i j}-\mu_{0 j} \neq 0$. Let $r_{i}=\left\{r_{i 1}, r_{i 2}, \ldots, r_{i k}\right\}$ be a rank vector where each rank value $r_{i j}$ is assigned to each deviation value $d_{i j}$ without regard to sign of $d_{i j}$. Let $W^{+}=\left(w_{1}{ }^{+}, w_{2}{ }^{+}, \ldots, w_{k}{ }^{+}\right)$be sum vector so that each sum value $w_{j}^{+}$is defined by equation 5 .

$$
w_{j}^{+}=\sum_{d_{i j}>0} r_{i j}
$$

The Hotelling's $T^{2}$ test is defined by equation 6 (Oja \& Randles, 2004, p. 598).

$$
T^{2}=n \bar{X}^{T} S^{-1} \bar{X}
$$

Where $\bar{X}$ and $S^{-1}$ are sample mean and sample covariance, respectively. 


$$
\begin{aligned}
& \bar{X}=\operatorname{ave}\left(X_{i}\right)=\frac{1}{n} \sum_{i=1}^{n} X_{i} \\
& S=\operatorname{ave}\left(\left(X_{i}-\bar{X}\right)\left(X_{i}-\bar{X}\right)^{T}\right)=\frac{1}{n-1} \sum_{i=1}^{n}\left(X_{i}-\bar{X}\right)\left(X_{i}-\bar{X}\right)^{T}
\end{aligned}
$$

The notation "ave" denotes average taken over all observation $1,2, \ldots, n$. The Hotelling's $T^{2}$ test is also asymptotically parametric test because $T^{2}$ approaches chi-squared distribution (Oja \& Randles, 2004, p. 599) when the sample is large enough and each $X_{i}$ conforms free distribution with zero mean and finite second moment (Oja \& Randles, 2004, p. 599).

$$
T^{2} \rightarrow \chi_{p}^{2}
$$

We will use chi-squared distribution to estimate LCL with $95 \%$ confidence degree in case that all $X_{i}$ are standardized so that their theoretical mean is zero.

$$
L C L=\chi_{p}^{2}(0.05)
$$

Where $\chi_{p}^{2}(0.05)$ is the lower $5 \%$ percentage of chi-squared distribution with $p$-degree of freedom.

\section{Discussions and Conclusions}

In general, NQC is combination of Wilcoxon rank-sum test and $\bar{X}$ control chart. The main point of MNQC method is to replace the deviation $d_{i}$ by the opposite of Pearson correlation between rating vector $X_{i}$ and the acceptable satisfied rating vector $\mu_{0}$ in calculating ranks. There are other researches solving the problem of non-parametric test in multivariate data.

As a convention, a test mentioned here is non-parametric test. Oja and Randles (Oja \& Randles, 2004 , p. 599) defined that a test $T$ satisfies affine invariant property if $T\left(D X_{1}, D X_{2}, \ldots, D X_{n}\right)=T\left(X_{1}\right.$, $\left.X_{2}, \ldots, X_{n}\right)$ where $D$ is a non-singular square matrix which is called transformation. Given vector $X$ $=\left(x_{1}, x_{2}, \ldots, x_{p}\right)^{T}$, Oja and Randles (Oja \& Randles, 2004, p. 599) defined the spatial sign function according to equation 8 .

$$
s(X)=\left\{\begin{array}{r}
|X|^{-1} X \text { if } X \neq \mathbf{0} \\
\mathbf{0} \text { if } X=\mathbf{0}
\end{array}\right.
$$

Where $\mathbf{0}=(0,0, \ldots, 0)^{T}$ is zero vector and $|X|$ is the length or module of vector $X$.

$$
|X|=\sqrt{\sum_{i=1}^{p} x_{i}^{2}}
$$

Note, $s(X)$ is a vector. Oja and Randles (Oja \& Randles, 2004, p. 599) defined the spatial sign for each observation $X_{i}$ based on spatial sign function according to equation 9 .

$$
S_{i}=s\left(A_{x} X_{i}\right)
$$

Of course, each spatial sign $S_{i}$ is a vector. The matrix $A_{x}$ is Tyler's transformation as seen in equation 10 .

$$
A_{x}^{T} A_{x}=V_{x}^{-1}
$$

Where $V_{x}$ is Tyler's shape matrix so that $V_{x}$ is positive definite symmetric $p_{x} p$ matrix and trace $\left(V_{x}\right)$ $=p$ with note that $p$ is dimension of $X_{i}$. Oja and Randles (Oja \& Randles, 2004, p. 599) defined sign mean $\bar{S}$ :

$$
\bar{S}=\operatorname{ave}\left(S_{i}\right)=\frac{1}{n} \sum_{i=1}^{n} S_{i}
$$


The matrix $V_{x}$ is calculated by an iterative process with note that $V$ is initialized $V=I_{p}$, as seen in equation 11 .

$$
V \leftarrow p V^{1 / 2} \text { ave }\left(S_{i} S_{i}^{T}\right) V^{1 / 2}
$$

When $\mid p$ ave $\left(S_{i} S_{i}^{T}\right)-I_{p} \mid$ is small enough, the process stops and $V_{x}=(p / \operatorname{trace}(V)) V$. Note, $I_{p}$ is unity matrix:

$$
I_{p}=\left(\begin{array}{cccc}
1 & 0 & \cdots & 0 \\
0 & 1 & \cdots & 0 \\
\vdots & \vdots & \ddots & \vdots \\
0 & 0 & \cdots & 1
\end{array}\right)
$$

Randles developed the multivariate sign test that rejects null hypothesis with large value of $Q^{2}$ as seen in equation 12 (Oja \& Randles, 2004, p. 599).

$$
Q^{2}=n p \bar{S}^{T} \bar{S}
$$

For large sample size, the test $Q^{2}$ with null hypothesis $H_{0}=\mathbf{0}$ approaches to chi-squared distribution (Oja \& Randles, 2004, p. 600):

$$
Q^{2} \rightarrow \chi_{p}^{2}
$$

In general, the essential point of the multivariate sign test of Oja and Randles is to define the special sign so that the Tyler's transformation $A_{x}$ derived from the iterative process in the equation 8 makes $Q^{2}$ to satisfy affine invariant property because $A_{x}$ makes the sign covariance matrix pave $\left(S_{i} S_{i}^{T}\right)$ equal or approach to $(1 / p) I_{p}$.

$$
\operatorname{pave}\left(S_{i} S_{i}^{T}\right)=\frac{1}{p} I_{p}
$$

For multivariate rank test, Oja and Randles replaced the spatial sign by the sign of transformed difference as seen in equation 13 (Oja \& Randles, 2004, p. 600):

$$
S_{i j}=s\left(A_{x}\left(X_{i}-X_{j}\right)\right)
$$

Note, $s($.$) is the spatial sign function specified by equation 8$. The centered rank is defined by equation 14 (Oja \& Randles, 2004, p. 600):

$$
R_{i}=\operatorname{ave}_{j}\left(S_{i j}\right)=\frac{1}{n} \sum_{i=1}^{n} S_{i j}
$$

The Tyler's transformation $A_{x}$ is determined via the Tyler's shape matrix $V_{x}$ specified by equation 10 but $V_{x}$ here is estimated by a different iterative process as seen in equation 15 .

$$
V \leftarrow \frac{p}{\operatorname{ave}\left(R_{i}^{T} R_{i}\right)} V^{1 / 2} \operatorname{ave}\left(R_{i}^{T} R_{i}\right) V^{1 / 2}
$$

Note, $V$ is initialized $V=I_{p}$. When $\left|\frac{p \text { ave }\left(R_{i} R_{i}^{T}\right)}{\text { ave }\left(\left|R_{i}\right|^{2}\right)}-I_{p}\right|$ is small enough, the process stops and $V_{x}=(p$ / trace $(V)) V$. Oja and Randles developed the multivariate signed-rank test that rejects null hypothesis with large value of $U^{2}$ as seen in equation 16 (Oja \& Randles, 2004, p. 599).

$$
U^{2}=\frac{n p}{4 \operatorname{ave}\left(\left|R_{i}\right|^{2}\right)}\left|\operatorname{ave}\left(s\left(A_{x}\left(X_{i}-X_{j}\right)\right)\right)\right|^{2}
$$

Where the average ave $\left(s\left(A_{x}\left(X_{i}-X_{j}\right)\right)\right)$ is over $i=1,2, \ldots, n$ and $j=1,2, \ldots, n$. For large sample size, the test $U^{2}$ with null hypothesis $H_{0}=\mathbf{0}$ approaches to chi-squared distribution (Oja \& Randles, 2004, p. 600):

$$
U^{2} \rightarrow \chi_{p}^{2}
$$


The preeminence of test methods of Oja and Randle is effective and solid. Computing Tyler's shape matrix $V_{x}$ needs iterative processes (Oja \& Randles, 2004, pp. 599-600) as seen in equations 10 and 14.

In general, the validity and effectiveness of the proposed MNQC method are not asserted because I do not make experiments on it. However, the ideology of MNQC with support of Pearson correlation is simple and reasonable. So, implementing MNQC method is surely easy. For further research, I will make experiment on MNQC and compare it with the test method of Oja and Randle.

\section{References}

Luong, N.-K., \& Nguyen, K.-T. T. (2013). Set of quality criteria for evaluating hospital quality. Hanoi: Vietnam Ministry of Health. Retrieved February 19, 2017

Oja, H., \& Randles, R. H. (2004). Multivariate Nonparametric Tests. (G. Casella, Ed.) Statistical Science, 19(4), 598-605. doi:10.1214/088342304000000558

Walpole, R. E., Myers, R. H., Myers, S. L., \& Ye, K. (2012). Probability \& Statistics for Engineers \& Scientists (9th ed.). (D. Lynch, Ed.) Boston, Massachusetts, USA: Pearson Education, Inc.

Wikipedia. (2017, February 10). 5S (methodology). (Wikimedia Foundation) Retrieved February 23, 2017, from Wikipedia website: https://en.wikipedia.org/wiki/5S_(methodology)

Wikipedia. (2017, February 21). Kaizen. (Wikimedia Foundation) Retrieved February 23, 2017, from Wikipedia website: https://en.wikipedia.org/wiki/Kaizen

Wikipedia. (2017, February 8). Performance indicator. (Wikimedia Foundation) Retrieved February 23, 2017, from Wikipedia website: https://en.wikipedia.org/wiki/Performance_indicator 